\title{
Electrical and optical spin injection in ferromagnet/semiconductor heterostructures
}

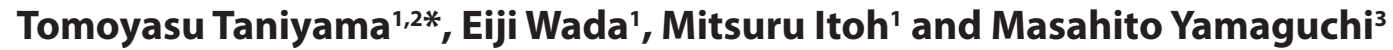 \\ Tokyo Institute of Technology and Nagoya University, Japan
}

Spin-based electronics or 'spintronics' is a rapidly expanding research area that offers the promise of surpassing the limits of conventional electrical charge-based semiconductor devices with potential advantages in speed and power consumption. For electron spin to be successfully employed in a spintronic device, the integration of electron charge and electron spin in a semiconductor is one of the central issues. In the basic concept of a spintronic device at present, the device functionality relies primarily on three different key processes: spin injection, spin manipulation and spin detection in ferromagnet/semiconductor heterostructures. This article reviews the current status of research on spin injection in semiconductors by both electrical and optical means and discusses the electron spin transport processes across the interface based on optical spin orientation.

$\mathrm{T}$ he operation of the rapidly expanding range of electronic devices, such as personal computers and mobile phones, is primarily based on the control of electron charge in semiconductors. Although the tremendous progress in microfabrication technologies has accelerated the miniaturization of electronic devices, the size of devices will soon encounter the fundamental physical limits of that miniaturization. Further scale reduction beyond these limits will require a radical alteration of the concept of functional devices. Control of the spin degree of freedom of an electron has brought about a new era of integration in electronics over the last ten years, and research in the field of 'spintronics' is currently being pursued extensively due to the potential of this approach for the development of a new direction in electronics. The field of spintronics spreads beyond the traditional boundaries among research fields, leading to interdisciplinary research that spans magnetism, semiconductors, photonics and electronics.

Since 1990, a number of spin-based device concepts have been proposed. Spin-based transistors [1-5], spin light-emitting diodes (spin-LEDs) [6,7] and spin transfer torque memories [8,9] are typical examples. One of the device concepts is depicted in Figure 1, where the device structure consists of a ferromagnetic source electrode, a highmobility semiconductor channel, a ferromagnetic drain electrode and a gate electrode. To operate a spin-based functional device, the primary issue is the efficient injection of spin-polarized electrons from the source ferromagnet into the semiconductor channel across the interface. The electron spin is then manipulated by the application of a voltage via the gate electrode [10], and the spin orientation is detected using a ferromagnetic drain electrode in a spin field-effect transistor (FET) or from the circular polarization of light emission in a spin-LED. Although the device concept is very simple and similar to that of a conventional FET, the implementation of spin-based devices is not that straightforward: the spin degree of freedom must be handled very delicately because the spin current rapidly depolarizes at the ferromagnet/semiconductor interface and the spin information will be lost within a time scale of several hundred picoseconds, even in a semiconductor $[11,12]$. This is in clear contrast to the conservation of electron charge. Therefore, there

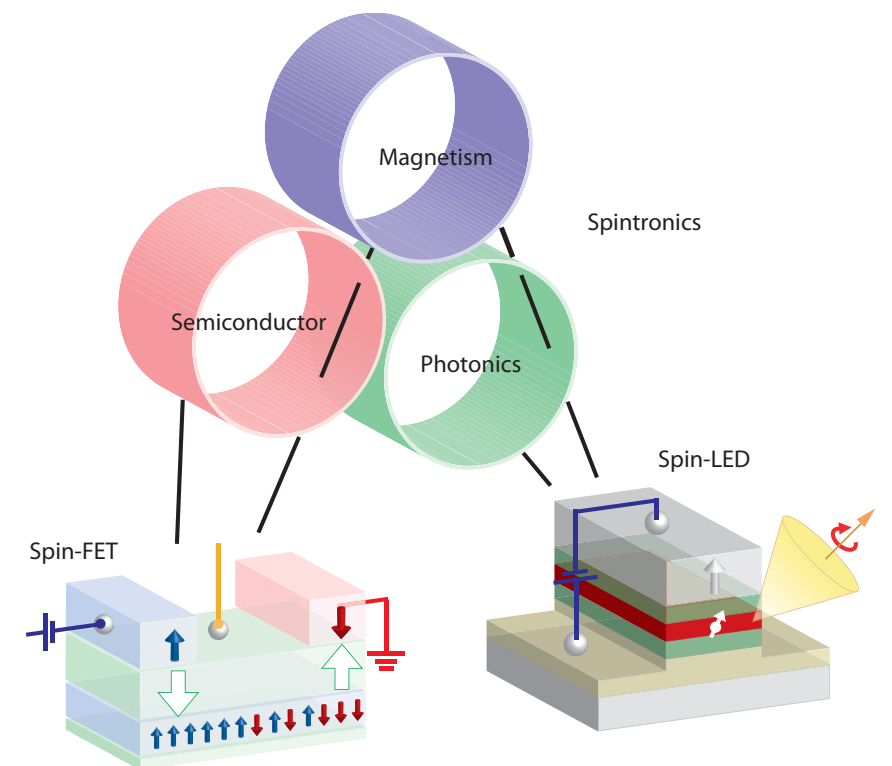

Figure 1. Conceptual illustration of spin-based electronic devices. In a spin-FET, spin-polarized electrons are injected from a source ferromagnet into a semiconductor and detected using a ferromagnetic drain electrode. In a spin-LED, circularly polarized light emission also occurs under spin injection.

are still obstacles to overcome in the implementation of such spintronic devices, and for this purpose a clear understanding of not only electron spin transport processes from a ferromagnet into a semiconductor, and vice versa, but also electron spin dynamics in semiconductors, is of fundamental and technological importance.

Our focus in this article is on reviewing the current status of this subject with special emphasis on electrical and optical spin injection

\footnotetext{
*Corresponding author. Email: taniyama.t.aa@m.titech.ac.jp

'Materials and Structures Laboratory, Tokyo Institute of Technology, 4259 Nagatsuta, Yokohama 226-8503, Japan

2PRESTO, Japan Science and Technology Agency, 3-5 Sanbancho, Chiyoda-ku, Tokyo 102-0075, Japan

3Department of Electronics, Nagoya University, Furo-cho, Chikusa-ku, Nagoya 464-8603, Japan
} 
into a semiconductor, discussing fundamental electron spin transport processes across ferromagnet/semiconductor interfaces. This review is organized as follows. In the next section, a brief summary of the mechanisms of the detection techniques for spin polarization is given. Techniques to quantify the spin polarization of electrons in semiconductor are critical requisites for the discussion of spin injection. To date, a number of experimental means have been introduced to detect spin polarization, including an optical approach relying on the optical transition selection rules in the radiative electron-hole recombination process in a semiconductor [13], an electrical approach using a nonlocal geometry of electrodes for detection $[14,15]$, the spin filtering effect [16] and magnetoresistance [17]. Spin relaxation mechanisms are also described in this section. That section is followed with a review of experimental work on electrical spin injection into a semiconductor, discussing the obstacles to electrical spin injection such as conductivity mismatch [18]. As the development of potential materials for the spin injection source is important for efficient spin injection, the materials used as spin sources, such as half-metallic materials [19-21] and ferromagnetic spin filters [22], are also summarized, and the effect of introducing an additional tunnel barrier on spin injection efficiency is discussed. In the third section, optical spin injection based on optical spin orientation is discussed $[11,23]$. As optical spin injection makes it possible to provide information about electron spin transport processes for developing electrical spin detection techniques, examples of the types of information that can be derived from experiments are presented in this section [24-26]. The review concludes with future prospects for spintronics technology.

\section{Detection of electron spin}

\section{Optical detection}

To study the issue of spin injection across a ferromagnet/semiconductor interface, the development of appropriate techniques for detecting electron spin polarization in semiconductor is of critical importance. In this section, we first review the optical approach for quantifying the spin polarization of electrons. The basic concept underlying the optical approach is analysis of the circular polarization of electroluminescence when spin-polarized electrons are injected into a III-V semiconductor quantum well $(\mathrm{QW})$ such as GaAs. In the direct bandgap semiconductor GaAs, the valence band splits into four-fold degenerate $P_{3 / 2}$ states, which consist of two-fold degenerate heavy-hole and light-hole subbands at $\Gamma_{8}$, and two-fold degenerate $P_{1 / 2}$ states at $\Gamma_{7}$ lying $\Delta=0.34 \mathrm{eV}$ below $P_{3 / 2}$, as shown in Figure 2(a) [27]. The conduction band, on the other hand, is two-fold degenerate $S_{1 / 2}$ at $\Gamma_{6}$. As spin-polarized electrons are injected into the conduction band, the electrons recombine with unpolarized holes in the valence band within recombination time $\tau_{\mathrm{R}}$, resulting in light emission. According to the optical transition selection rules, two transition processes from $S_{1 / 2}$ to $P_{3 / 2}$ are allowed, where the transition processes give rise to light emissions with right- or left-handed circular polarization [11]. The transition probabilities to the heavy-hole subband and the light-hole subband, however, are different; the transition probability to the light-hole subband $\left(m_{j}= \pm 3 / 2\right)$ is a factor of three larger than those for the heavy-hole subbands $\left(m_{j}= \pm 1 / 2\right)$, leading to light with a maximum circular polarization of $50 \%$ given an injection of fully spin-polarized electrons (Figure 2(b)). Therefore, the close link between the spin polarization $P_{\mathrm{S}}$ of electrons injected into the conduction band and the circular polarization $P_{\text {circ }}$ of light emission through radiative recombination offers a quantitative measure of spin polarization using the relation $2 P_{\text {circ }}=P_{\mathrm{S}}$. This approach can be used to quantify the efficiency of spin injection across the ferromagnet/semiconductor interface.

It should also be noted that the spin polarization obtained using the optical approach provides the lower bound because the spin depolarizes in the semiconductor due to spin relaxations at the rate of the spin relaxation time $\tau_{\mathrm{s}}$ until recombination. There are several types of spin relaxation mechanisms, as any fluctuation or inhomogeneity of spin interaction causes spin relaxation. However, in III-V semiconductors a
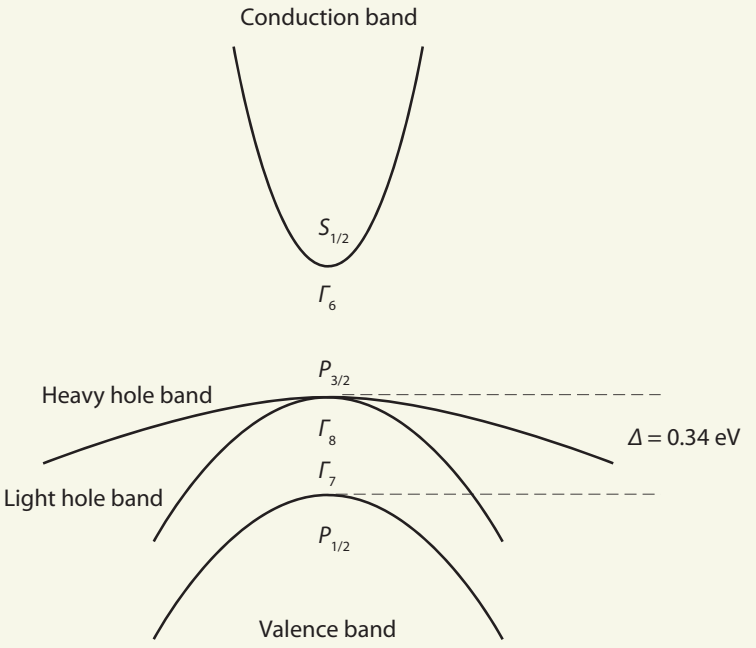

b Polarized light emission

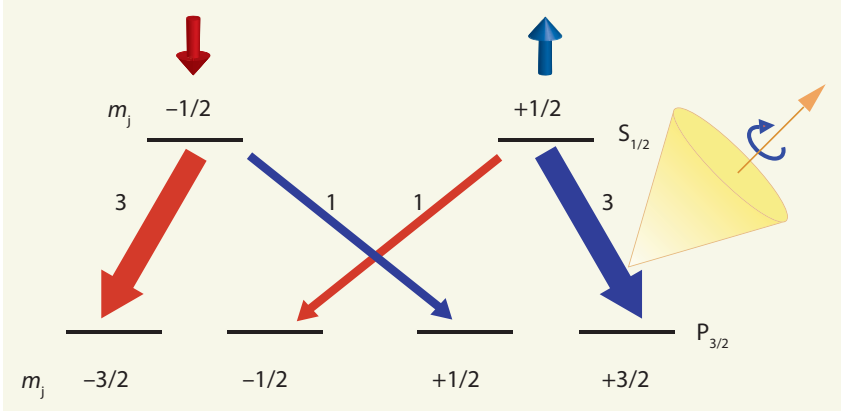

c

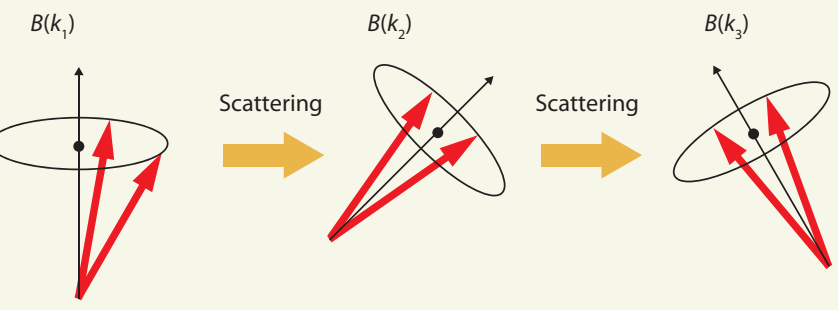

Figure 2. (a) Band diagram for GaAs. (b) Radiative recombination of spin-up and spin-down electrons according to optical transition selection rules. (c) Schematic diagrams of the D'yakonov-Perel'spin relaxation mechanism.

such as GaAs, a few relevant mechanisms dominate the spin relaxation: the D'yakonov-Perel' mechanism, which originates from the spin splitting of the conduction band due to the inversion symmetry breaking of a crystal [28]; the Bir-Aronov-Pikus mechanism due to the exchange interaction between electrons and holes [29]; the ElliottYafet mechanism caused by momentum relaxation through spin-orbit coupling [30,31]; and a hyperfine interaction mechanism arising from the magnetic interaction between electron spin and nuclear spin [32].

The spin relaxation mechanisms have been discussed in a recent review [12]. As an example of these mechanisms, the D'yakonov-Perel' mechanism is described here in detail. In a crystal lacking inversion symmetry, such as III-V (GaAs) and II-IV ( $\mathrm{ZnSe})$ semiconductors, the D'yakonov-Perel' mechanism dominates spin relaxation. In general, the momentum states with $\boldsymbol{k}$ and $\boldsymbol{- k}$ are not degenerate without inversion symmetry $\left(E_{k} \neq E_{-k}\right)$. According to Kramer's theorem, on the other hand, $E_{k \uparrow}=E_{-k \downarrow}$, hence the relationship $E_{k \uparrow} \neq E_{k \downarrow}$ is fulfilled. This spin splitting of the momentum states can be regarded as 'Zeeman 
splitting' in the presence of a $\boldsymbol{k}$-dependent effective magnetic field $\boldsymbol{B}(\boldsymbol{k})$, about which electron spin precesses with a Larmor frequency of $\boldsymbol{\Omega}(\boldsymbol{k})=(e / m) \boldsymbol{B}(\boldsymbol{k})$. Now consider that the momentum of an electron changes randomly due to momentum scattering events at an interval of the momentum scattering time $\tau_{\mathrm{p}}$. The electron spin precesses about the effective magnetic field between the scattering events, and after the scattering the electron spin precesses about a different effective magnetic field (Figure 2(c)). This random walk evolution of spin phase causes spin relaxation. As the momentum scattering event and the Larmor precession play a key role in this mechanism, two regimes for the spin relaxation can be considered: (1) $\tau_{\mathrm{p}} \Omega<<1$ and (2) $\tau_{\mathrm{p}} \Omega \geq 1$. Regime (1) describes the spin precession about random magnetic fields fluctuating with a time interval of $\tau_{\mathrm{p}}$. Because individual electron spins do not precess a full cycle within $\tau_{\mathrm{p}}$, the spin phase follows the random walk and motional narrowing occurs accordingly. Thus, the spin relaxation rate $1 / \tau_{\mathrm{s}}=\Omega^{2} \tau_{\mathrm{p}}$, that is, stronger scattering leads to slow spin dephasing. In regime (2), on the other hand, electron spins experience a full cycle of precession before the adjacent scattering event, and the spin dephasing rate is given by $1 / \tau_{\mathrm{s}} \approx \Delta \Omega$, where $\Delta \Omega$ is the distribution width of $\Omega$. Since the spin dephasing in regime (1) is associated with spin-independent scattering with impurities and phonons, the D'yakonov-Perel' spin relaxation mechanism depends strongly on the doping concentration and temperature.

Taking spin relaxation into account, the circular polarization $P_{\text {circ }}$ can be written according to a simple rate equation [33]:

$$
2 P_{\text {circ }}=P_{\mathrm{S}}=\frac{P_{\mathrm{S}}^{0}+\tau_{\mathrm{R}} / 2 \tau_{\mathrm{S}}(1-v)}{1+\tau_{\mathrm{R}} / 2 \tau_{\mathrm{S}}(1+v)}
$$

where $P_{S}{ }^{0}$ denotes the spin polarization just after the electrons are injected into the semiconductor and $v$ is the ratio of the electron populations between the upper and lower Zeeman levels given by the FermiDirac distribution. If the spin relaxation time is much shorter than the recombination time, Equation (1) is reduced to $2 P_{\text {circ }}=(1-v) /(1+v)$. Also, if the Zeeman polarization is vanishing, that is, $v=1$, then the circular polarization results in

$$
2 P_{\text {circ }}=P_{\mathrm{S}}=\frac{P_{\mathrm{S}}^{0}}{1+\tau_{\mathrm{R}} / \tau_{\mathrm{S}}}
$$

revealing that the circular polarization depends on the value of $\tau_{\mathrm{R}} / \tau_{\mathrm{S}}$ and could show a temperature variation accordingly.

\section{Electrical detection}

Another approach for measuring electron spin injected into semiconductors is to use a device structure with a non-local geometry of electrodes as depicted in Figure 3(a). The non-local detection of electron spin was first demonstrated in a metallic lateral spin valve structure by Johnson and Silsbee [15]. Jedema et al. [14] also employed an $\mathrm{NiFe} / \mathrm{Cu}$ junction structure, where electrons flow from the ferromagnetic NiFe electrode (FM1) into the nonmagnetic copper electrode (NM), and the voltage between another NiFe electrode (FM2) and the other side of the copper electrode is measured. As there is no current flow in the area between the voltage electrodes, no voltage signal would occur in a traditional manner. However, as spin-polarized electrons are injected into the copper electrode, a splitting of the electrochemical potentials $\mu^{\uparrow \downarrow}$, which are the sum of the electrical potential of the conduction band edge and the chemical potential for spin-up and spin-down electrons, appears in the copper electrode close to the $\mathrm{NiFe} / \mathrm{Cu}$ junction, causing a spin-dependent potential difference between the voltage electrodes (Figure 3(b)). The difference in the spin-split electrochemical potentials is termed spin accumulation, and the technique for measuring spin accumulation is called non-local detection because the voltage electrodes detect the spindependent potential difference non-locally in this structure.

Let us consider the mechanism of the spin-split electrochemical potentials in the copper electrode in more detail. This effect primarily a

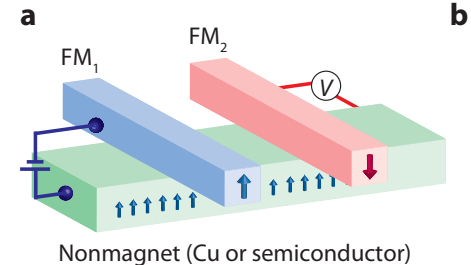

b

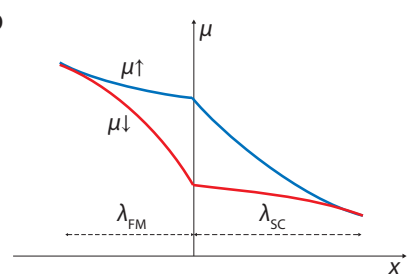

C

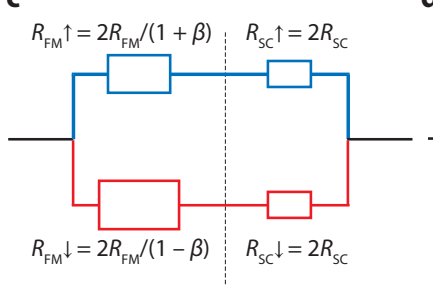

d

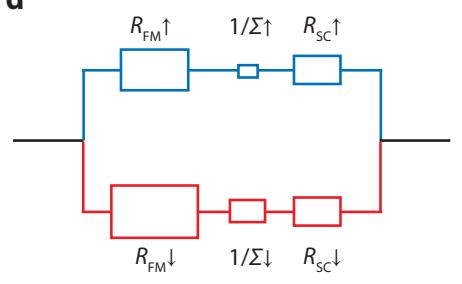

Figure 3. (a) A non-local geometry of electrodes for detecting spin accumulation in a nonmagnet. (b) Spin-dependent electrochemical potential. Spin accumulation occurs near the ferromagnet/nonmagnet interface. $(c, d)$ Two current resistor models for a ferromagnet/semiconductor interface without (c) and with (d) a spin-selective insulating interlayer between the ferromagnet and the semiconductor.

arises from the difference in the spin-dependent conductances of a ferromagnetic material and a nonmagnetic material, as first formulated by van Son et al. [34] and Johnson et al. [35]. If the electron transport can be treated in the diffusive regime and the momentum scattering length for electrons is much shorter than the spin diffusion length (a measure of how long electron spins traverse in a material without losing spin information), the electrochemical potentials for spin-up and spindown electrons are defined simply as follows. Ohm's law for the two spin channels can be written as

$$
\nabla \mu^{\uparrow \downarrow}=-\frac{e j^{\dagger \downarrow}}{\sigma^{\uparrow \downarrow}}
$$

where $\sigma^{\uparrow \downarrow}$ are the conductivities for spin-up and spin-down channels, $j^{\dagger \downarrow}$ are the current densities for spin-up and spin-down electrons, and $e$ is the electron charge. The total current density including both $j^{\dagger \downarrow}$ should be continuous at the interface, as given by

$$
\nabla\left(j^{\uparrow}+j^{\downarrow}\right)=0
$$

The interaction between both spin channels, that is, spin flipping, should also be considered in the formalism as given by the following diffusion equation:

$$
\frac{\mu^{\uparrow}-\mu^{\downarrow}}{\tau_{\mathrm{s}}}=D \nabla\left(\mu^{\uparrow}-\mu^{\downarrow}\right)
$$

where $D$ is the weighted diffusion constant for both spin electrons. These equations can be solved within the boundary conditions that $\mu^{\uparrow \downarrow}$ are continuous at the ferromagnet/nonmagnet interface. Consequently, splitting of the spin-dependent electrochemical potentials or spin accumulation arises when a current flows across the interface as shown in Figure 3(b). We will see spin accumulation later again in a more intuitive manner using a two current resistor model.

The non-local electrical detection technique can be applied directly to measure spin accumulation in a semiconductor [36-41]. The non-local electrical detection of spin accumulation in GaAs was first demonstrated in 2007 by Lou et al. [37] using an Fe/GaAs(001) heterostructure with a lightly $\mathrm{n}$-doped GaAs epilayer. The spin diffusion length was estimated to be $6 \mu \mathrm{m}$ at $50 \mathrm{~K}$. Spin injection into silicon was also demonstrated in the same year by Appelbaum et al. [38] and van't Erve et al. [39]. Room-temperature detection of spin accumulation in silicon was then demonstrated in 2009 by Dash et al. [40] 
using a lateral three-terminal geometry of $\mathrm{NiFe} / \mathrm{Al}_{2} \mathrm{O}_{3} / \mathrm{Si}$ electrodes. This result was followed by a more convincing observation of non-local spin signals in an $\mathrm{Fe} / \mathrm{MgO} / \mathrm{Si}$ device with the non-local geometry of electrodes by Sasaki et al. [41].

Another electrical spin detection technique is based on spindependent electron transport from a semiconductor into a ferromagnet. To examine the principle of electrical spin detection, it is helpful to review the transport processes of electron spin across a ferromagnet/ semiconductor interface. Consider the generation of spin-polarized electrons in GaAs, as shown in Figure 4(a). If a forward electric bias voltage $V_{\mathrm{B}}$ is applied at the interface, the generated electrons propagate toward the ferromagnetic layer due to the difference in the electrochemical potentials of the ferromagnetic layer and the semiconductor. Once the electrons reach the interface, the electrons transmit across the metal/semiconductor Schottky barrier via a tunneling process. However, the transmission probability across the interface depends on the relative orientation between the spin of the generated electrons and the magnetization direction of the ferromagnet, as the conductances of the spin-up and spin-down channels in the ferromagnet are proportional to the density of states for each spin channel via the Einstein relation. In this case, it is clear that a significant spin dependence of the electron transmission arises. This spin-dependent effect of electron transmission is commonly referred to as spin filtering because the ferromagnet filters one of the spin-up or spin-down electrons at the interface [16]. Experimentally, the spin filtering effect can be detected by measuring the spin-dependent photocurrent across the interface, which will be discussed in detail in the following section. The spin dependence of electron transmission is very sensitive to the spin-dependent density of states at the interface as well as the quality of the interface. So far, the spin-dependent density of states at the interface is not that robust nor well-defined, so the spin filtering effect does not seem appropriate for the quantitative characterization of spin polarization, although it can be used to discuss the qualitative spin polarization. It should also be noted that spin-polarized electrons injected into a semiconductor can be detected by measuring magnetoresistance in a ferromagnet/semiconductor/ferromagnet structure, where one ferromagnetic layer is a spin injector and the other works as a spin detector [17].

\section{Electrical spin injection into semiconductors}

\section{Experimental demonstrations of electrical spin injection}

As discussed above, to date two types of spin detection techniques, optical detection and electrical detection, have been used successfully. Electrical detection, however, cannot offer a quantitative and modelindependent estimate of spin polarization; a model fitting to the electrical signals provides an estimate of spin polarization. Optical detection, on the other hand, can quantify the spin polarization directly using a very simple scheme. Therefore, we hereafter focus on the optical detection of spin polarization in a semiconductor to discuss spin injection efficiency. The optical detection of an electrically injected spin polarization in a semiconductor was first demonstrated in 1999 independently by Ohno et al. [7] and Fiederling et al. [6], who used LED structures with InGaAs or GaAs QWs and analyzed the optical polarization of electroluminescence as discussed in the previous section (Figure 4(b)). Ohno et al. used the ferromagnetic semiconductor GaMnAs as the spin injection source, whereas Fiederling et al. employed a BeMnZnSe spin aligner. Since the first demonstrations, an enormous number of studies on similar systems have been reported, in which the QW structures are modified and other spin injection sources are used [42-64].

To achieve high efficiency of spin injection at room temperature, ferromagnetic injection sources with a Curie temperature far above room temperature are necessary. Most research in this area has therefore focused on the use of ferromagnetic metals such as iron and Heusler alloys rather than ferromagnetic semiconductors. For example, Zhu and co-workers [42] demonstrated the injection of spin-polarized electrons from an iron layer into an $\mathrm{InGaAs} \mathrm{QW}$ at room temperature by a

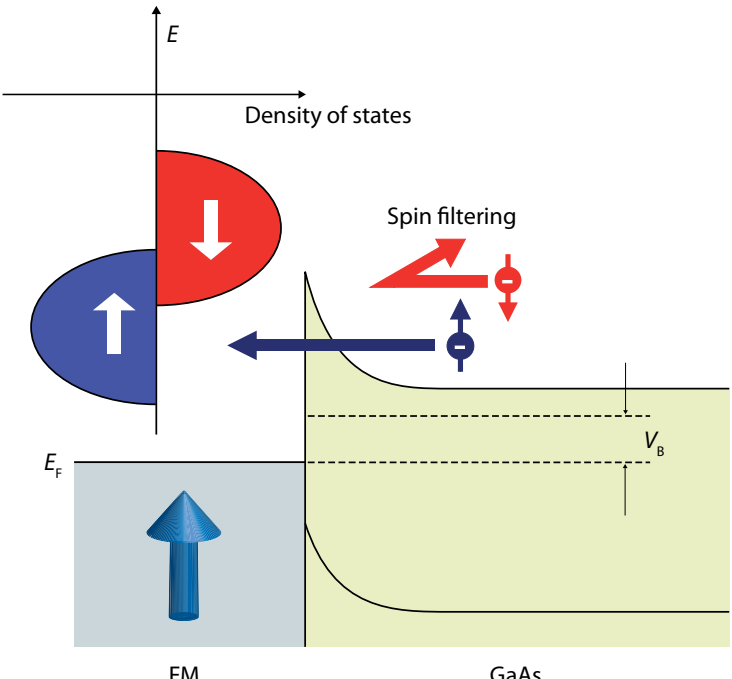

b

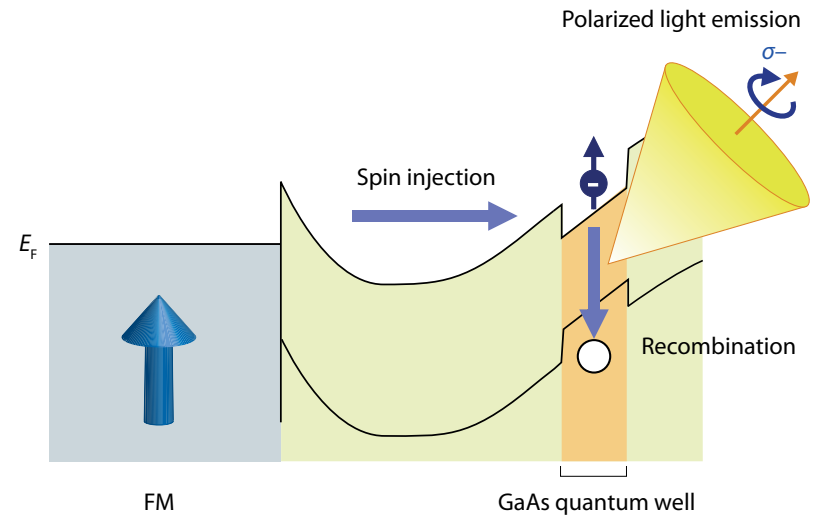

Figure 4. (a) Schematic diagram of the spin filtering effect at a ferromagnet/semiconductor interface. Spin-up electrons transmit across the ferromagnet/semiconductor interface, while spin-down electrons are reflected by the interface. (b) Schematic band diagram of a ferromagnetic metal/semiconductor QW heterostructure with circularly polarized light emission.

extracting the light emissions arising from the transitions to the lighthole and heavy-hole subbands separately, achieving spin polarization of $2 \%$ in the quantum well. Hanbicki et al. [44] obtained a large spin polarization of $32 \%$ at $4.5 \mathrm{~K}$ by injecting electrons from iron into GaAs across an Fe/AlGaAs Schottky interface. The same group also decomposed the electroluminescence spectra for a similar spin-LED structure. The spectra were found to include excitonic features and the replica of excitons associated with the emission of zone-edge phonons.

Electrical spin injection into silicon has attracted particular interest for the possibility of incorporating silicon-based spintronic devices into the current production technologies for semiconductor electronics. Jonker et al. $[64,65]$ made the first attempt to inject spin-polarized electrons into a silicon spin-LED structure. Measurements of spin polarization using the optical detection technique revealed a spin polarization of $10 \%$, which although relatively low could be enhanced by improving the injection of electron spins into silicon. A number of studies have recently been conducted in this area [38-41].

As the optical detection of electron spin is based on the conversion of out-of-plane electron spins into the photon helicity of light emission, magnetic spin sources must be saturated normal to the layer plane. This generally requires the application of a high magnetic field of over $2 \mathrm{~T}$. To observe spin polarization in the low-field region, Motsnyi et al. [63] measured the spin polarization of electrons by an ingenious approach that relies on the oblique Hanle effect. In the measurement geometry, a magnetic field low enough to align the magnetization 
in-plane is applied at an angle of $45^{\circ}$ with respect to the plane normal. When spin-polarized electrons are injected from the in-plane magnetized ferromagnetic film, the electron spins precess about the applied magnetic field in the semiconductor at the Larmor frequency, giving the average out-of-plane component of the electron spin in the semiconductor. Therefore, even in a low magnetic field, the out-of-plane component contributes to the circular polarization of light emission, which makes it possible to estimate the efficiency of spin injection optically. In this way, Motsnyi et al. estimated the spin polarization for a $\mathrm{CoFe} / \mathrm{AlO}_{x}$ spin injector to be $9.2 \%$ at $80 \mathrm{~K}$. Besides the oblique Hanle geometry, the use of perpendicularly magnetized films is also a potentially effective approach for the optical detection of spin polarization in the low-field regime. Using out-of-plane magnetized FePt spin injection sources, Sinsarp et al. [51] obtained a circular polarization of $1.5 \%$ at remanence and $11.5 \%$ in a perpendicular magnetic field of $1 \mathrm{~T}$ at room temperature.

\section{Obstacles to electrical spin injection}

Achieving a high efficiency of spin injection from a ferromagnet into a semiconductor is a necessary step in the development of spintronic devices. There is, however, a fundamental obstacle to obtaining efficient spin injection into a semiconductor via a diffusive process, as suggested by Schmidt et al. $[18,66]$. We discuss the obstacles to electron spin injection into a semiconductor using a two current resistor model, which is equivalent to the theory of spin accumulation introduced in the previous section. Assume in a first approximation that spin-up and spin-down electrons flow in different channels independently across a ferromagnet/semiconductor interface and that the total current can be given by the parallel circuit of the spin-dependent channels when the current flows as shown in Figure 3(c). In this parallel circuit, the resistances of both channels in the spin-unpolarized semiconductor are $2 R_{\mathrm{SC}}$, while the resistances of the ferromagnet for spin-up and spin-down channels $R_{\mathrm{FM}}^{\uparrow \downarrow}$ are given by

$$
R_{\mathrm{FM}}^{\uparrow \downarrow}=\frac{2 R_{\mathrm{FM}}}{1 \pm \beta}
$$

using a bulk spin polarization $\beta=\left(\sigma^{\uparrow}-\sigma^{\downarrow}\right) /\left(\sigma^{\uparrow}+\sigma^{\downarrow}\right)$, where $R_{\mathrm{SC}}$ and $R_{\mathrm{FM}}$ are the total resistances of the semiconductor and the ferromagnet, respectively, and $\sigma^{\uparrow \downarrow}$ is the conductivity of the ferromagnet for each spin channel. Therefore, a simple calculation gives the current polarization $\gamma=\left(j^{\uparrow}-j^{\downarrow}\right) /\left(j^{\dagger}+j^{\downarrow}\right)$ in the device as

$$
\gamma=\beta \frac{R_{\mathrm{FM}}}{R_{\mathrm{SC}}} \frac{1}{R_{\mathrm{FM}} / R_{\mathrm{SC}}+\left(1-\beta^{2}\right)}
$$

In the approximation above, we have neglected spin flip processes, and have calculated the resistance over the entire sample along the flow of current. However, if spin flip processes are included, the spin-up and spin-down electrons are mixed and the two current resistor model should be applied only to the region within the spin diffusion length $\lambda=\sqrt{D \tau_{\mathrm{s}}}$ near the interface. Equivalently, the splitting of the electrochemical potentials for the spin-up and spin-down channels remains only in the region within the spin diffusion length. Thus, the resistances of the semiconductor $R_{\mathrm{SC}}$ and the ferromagnet $R_{\mathrm{FM}}$ should be substituted by $\lambda_{\mathrm{SC}} / \sigma_{\mathrm{SC}}$ and $\lambda_{\mathrm{FM}} / \sigma_{\mathrm{FM}}$, respectively, as given by

$$
\gamma=\beta \frac{\lambda_{\mathrm{FM}}}{\sigma_{\mathrm{FM}}} \frac{\sigma_{\mathrm{SC}}}{\lambda_{\mathrm{SC}}} \frac{1}{\left(\lambda_{\mathrm{FM}} \sigma_{\mathrm{SC}}\right) /\left(\lambda_{\mathrm{SC}} \sigma_{\mathrm{FM}}\right)+\left(1-\beta^{2}\right)}
$$

where $\sigma_{\mathrm{FM}(\mathrm{SC})}$ and $\lambda_{\mathrm{FM}(\mathrm{SC})}$ are the conductivity and spin diffusion length of the ferromagnet (semiconductor), respectively. It should be noted that Equation (8) can also be obtained by solving Equations (3)-(5) directly. The important consequence from the description above is that $R_{\mathrm{FM}} / R_{\mathrm{SC}}$ dominates the current spin polarization if the resistance of the ferromagnet is much smaller than that of the semiconductor, whereas current spin polarization in the semiconductor is proportional to the bulk spin polarization of the ferromagnet. In other words, spin injection from a ferromagnetic metal into a semiconductor, where $\sigma_{\mathrm{SC}} / \sigma_{\mathrm{FM}} \approx 10^{-4}$ is fulfilled, is difficult in principle as an inherent limitation of the diffusive process of electron spin transmission across the ferromagnetic metal/semiconductor interface. This fundamental obstacle is obviously due to the conductivity mismatch between the ferromagnetic metal and the semiconductor.

To circumvent this conductivity mismatch, Rashba [67] proposed the introduction of an additional spin-dependent tunnel barrier between the ferromagnet and the semiconductor, suggesting that current spin polarization is governed by the electrical properties of the barrier as shown by the equivalent circuit in Figure 3(d). From this figure, the current spin polarization can be obtained as

$$
\begin{aligned}
\gamma & =\frac{2 R_{\mathrm{FM}} /(1-\beta)-2 R_{\mathrm{FM}} /(1+\beta)+1 / \Sigma_{\downarrow}-1 / \Sigma_{\uparrow}}{2 R_{\mathrm{FM}} /(1-\beta)+2 R_{\mathrm{FM}} /(1+\beta)+1 / \Sigma_{\downarrow}+1 / \Sigma_{\uparrow}+4 R_{\mathrm{SC}}} \\
& =\beta \frac{r_{\mathrm{F}}}{r_{\mathrm{F}}+r_{\mathrm{N}}+r_{\mathrm{C}}}+\frac{1 / \Sigma_{\downarrow}-1 / \Sigma_{\uparrow}}{1 / \Sigma_{\downarrow}+1 / \Sigma_{\uparrow}} \frac{r_{\mathrm{C}}}{r_{\mathrm{F}}+r_{\mathrm{N}}+r_{\mathrm{C}}}
\end{aligned}
$$

where $1 / \Sigma^{\uparrow \downarrow}$ are the tunnel barrier contact resistances, $r_{\mathrm{F}}=\lambda_{\mathrm{FM}}\left(\sigma^{\uparrow}+\sigma^{\downarrow}\right) /\left(4 \sigma^{\uparrow} \sigma^{\downarrow}\right), r_{\mathrm{SC}}=\lambda_{\mathrm{SC}} / \sigma_{\mathrm{SC}}$ and $r_{\mathrm{C}}=\left(\Sigma^{\uparrow}+\Sigma^{\downarrow}\right) /\left(4 \Sigma^{\dagger} \Sigma^{\downarrow}\right)$. As the ferromagnet is a metal and the contact resistance is large $\left(r_{\mathrm{C}}>r_{\mathrm{SC}}, r_{\mathrm{FM}}\right)$, the first term is negligible and the second term reduces to $\left(\Sigma^{\uparrow}-\Sigma^{\downarrow}\right) /\left(\Sigma^{\uparrow}+\Sigma^{\downarrow}\right)$, dominating the current spin polarization in this case. Therefore, the introduction of a spin-selective barrier contact could be a very effective method for achieving high efficiency of spin injection.

\section{Source materials for spin injection}

As discussed in the previous sections, the use of half-metallic materials such as Heusler alloys $\left(\mathrm{Co}_{2} \mathrm{MnGe}[58], \mathrm{Co}_{2} \mathrm{MnGa}\right.$ [61,62], $\mathrm{Fe}_{3} \mathrm{Si}$ [33]) and magnetic oxides $\left(\mathrm{La}_{0.7} \mathrm{Sr}_{0.3} \mathrm{MnO}_{3}, \mathrm{CrO}_{2}, \mathrm{Fe}_{3} \mathrm{O}_{4}[21]\right)$ as spin injection sources is a promising means of enhancing the efficiency of spin injection [33,58-62]. Kawaharazuka et al. [33] reported on spin injection from the Heusler alloy $\mathrm{Fe}_{3} \mathrm{Si}$ into an $\mathrm{InGaAs} / \mathrm{GaAs}$ QW structure, which achieved a circular polarization of $3 \%$ at $25 \mathrm{~K}$. Dong et al. [58] used a Heusler alloy $\mathrm{Co}_{2} \mathrm{MnGe}$ as a spin source to inject spin-polarized electrons into an $\mathrm{Al}_{0.1} \mathrm{Ga}_{0.9} \mathrm{As} / \mathrm{GaAs} \mathrm{QW}$, affording an estimated spin polarization of $27 \%$ at $2 \mathrm{~K}$. Damsgaard et al. [62] reported a spin polarization of $6.4 \%$ at $5 \mathrm{~K}$ for a $\mathrm{Co}_{2} \mathrm{MnG}$ a spin aligner. Despite the prediction of larger spin polarization, to date the highest spin polarization reported remains relatively low, attributed variously to the properties of the interface, such as inhomogeneities, defects, strain and reduced symmetries, and the effect of finite temperature on the electronic, magnonic and phononic states at the interface [68-70]. Magnons generated by charge carriers crossing the interface may destroy the complete spin polarization, as discussed in the context of tunnel magnetoresistance devices.

The introduction of a thin insulating barrier layer between the ferromagnetic metal and the semiconductor is one approach to enhancing the efficiency of spin injection. The use of a $\mathrm{CoFe} / \mathrm{MgO}(001)$ interface, where $\mathrm{MgO}$ had previously been demonstrated to act as a spin aligner in tunnel magnetoresistive devices, was reported by Jiang et al. [56] to achieve spin polarization of as high as $57 \%$ at $100 \mathrm{~K}$, and $47 \%$ at room temperature. Manago et al. [52] reported on spin injection into a GaAs QW using a ferromagnetic metal/ $\mathrm{Al}_{2} \mathrm{O}_{3}$ spin source at room temperature, and van't Erve and co-workers [54] obtained a relatively large spin polarization of $40 \%$ at $5 \mathrm{~K}$ by introducing an $\mathrm{Al}_{2} \mathrm{O}_{3}$ layer between the iron and AlGaAs layers. Recently, the effect of an $\mathrm{Fe} / \mathrm{GaO}_{x}$ tunnel injector on spin injection efficiency was studied by Saito et al. [55], who also achieved a spin polarization of $40 \%$ at $2 \mathrm{~K}$.

A spin-selective insulating interlayer, which acts as a spin filter, can also be used to enhance the efficiency of spin injection into a semiconductor $[22,24,25,71-73]$. Wada et al. [24,25] recently reported the spin 

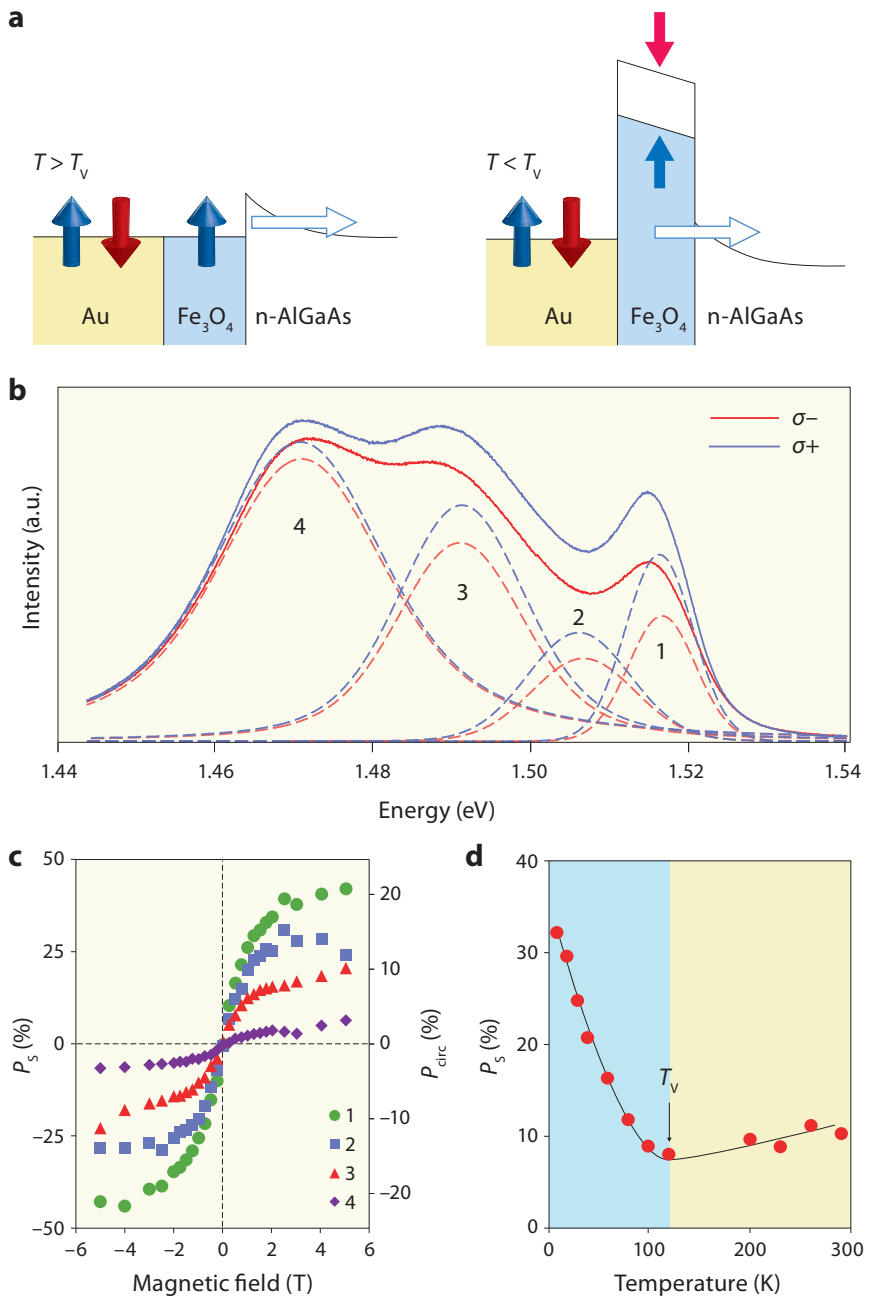

Figure 5. (a) Mechanisms of spin injection across an $\mathrm{Fe}_{3} \mathrm{O}_{4}$ layer below and above the Verwey transition temperature. (b) Circularly polarized electroluminescence spectra for an $\mathrm{Fe}_{3} \mathrm{O}_{4} / \mathrm{GaAs}$ QW spin-LED structure. (c) Magnetic field dependence of the circular polarization and spin polarization of electroluminescence for sub-features 1-4. (d) Temperature variation in spin polarization. A distinct change in spin polarization occurs at the Verwey transition of $\mathrm{Fe}_{3} \mathrm{O}_{4}$. Modified after Refs 24 and 25 (@ 2010 AIP).

polarization of electrons injected into an $\mathrm{AlGaAs} / \mathrm{GaAs} \mathrm{QW}$ across a thin $\mathrm{Fe}_{3} \mathrm{O}_{4}$ layer to investigate the effect of a magnetic insulating layer on spin injection efficiency. $\mathrm{Fe}_{3} \mathrm{O}_{4}$ has a ferrimagnetic structure with a high theoretically predicted spin polarization at room temperature [21]. Another important physical aspect of $\mathrm{Fe}_{3} \mathrm{O}_{4}$ is its charge-ordering Verwey transition, which occurs at $120 \mathrm{~K}$. In the low-temperature charge-ordered phase, $\mathrm{Fe}^{2+}$ and $\mathrm{Fe}^{3+}$ ions form an ordered arrangement and the material possesses an insulating electrical property. Consider what occurs if a thin $\mathrm{Fe}_{3} \mathrm{O}_{4}$ layer is introduced as a spin injector. While efficient spin injection may be realized at room temperature due to its high spin polarization, the mechanism of spin injection would change across the Verwey transition. The spin injection process from a metallic $\mathrm{Fe}_{3} \mathrm{O}_{4}$ above the Verwey transition temperature and that across the magnetic insulating $\mathrm{Fe}_{3} \mathrm{O}_{4}$ below that temperature are illustrated in Figure 5(a). Attempts have been made to confirm this scenario experimentally. Figure 5(b) shows a typical electroluminescence spectrum, which can be decomposed into four sub-features. Feature 1 is due to light emission from the GaAs QW, whereas features 2, 3 and 4 originate from the luminescence due to free exciton recombination, the conduction band to acceptor transition, and the longitudinal optical phonon replica of feature 2 in the GaAs bulk, respectively [46]. All the decomposed sub-features show a field dependence and the saturation value of the spin polarization for feature 1 is over $40 \%$ at $10 \mathrm{~K}$ (Figure 5(c)). This large spin polarization is likely associated with electron spin transport across the magnetic insulating $\mathrm{Fe}_{3} \mathrm{O}_{4}$ layer. Alteration of the mechanism of spin injection across the $\mathrm{Fe}_{3} \mathrm{O}_{4}$ layer manifests itself in the temperature variation of the spin polarization of electrons, as shown in Figure 5(d). Spin polarization above $120 \mathrm{~K}$ decreases slightly with decreasing temperature, whereas the temperature variation below $120 \mathrm{~K}$ is much more prominent. The temperature at which a distinct change in the temperature dependence can be seen corresponds to the Verwey transition of $\mathrm{Fe}_{3} \mathrm{O}_{4}$, indicating that the use of a spin-selective insulating interlayer is feasible for achieving high efficiency of spin injection into a semiconductor. We also note here that the observations of a distinct change in spin polarization are not caused by replacing materials but by alteration of the electronic properties of the same material, providing strong support for Rashba's suggestions for efficient spin injection as discussed in the previous section.

\section{Optical spin injection and spin transport}

Optical spin orientation and spin-dependent photocurrent

Besides electrical spin injection, an optical approach based on optical spin orientation is also used to generate spin-polarized electrons in III-V semiconductors. Optical spin orientation was first demonstrated in 1968 by Lampel [74]. Pierce and Meier [23] subsequently developed an approach for optically detecting spin-oriented electrons emitted from GaAs using a Mott detector. The fundamental mechanism underlying optical spin orientation is relatively simple, being based on the inverse electron transition process to that for the radiative recombination of electrons and holes in III-V semiconductors [11]. When GaAs is irradiated with right- or left-handed circularly polarized light at the bandgap energy, spin-up or spin-down electrons with a maximum spin polarization of $50 \%$ are generated according to optical transition selection rules. These processes are the precise inverse of those given in Figure 2(b). An advantage of this sophisticated approach is that it makes it possible to generate spin-polarized electrons in GaAs irrespective of the electronic features of the interface, in contract to the case of electrical spin injection. Therefore, the use of optical spin orientation provides a basis for studying electron spin transport from a semiconductor into a ferromagnet across the interface. As spin-polarized electrons are generated this way in the GaAs of a ferromagnetic metal/GaAs heterostructure, the electrons travel toward the ferromagnetic metal/GaAs Schottky interface under forward bias and traverse the interface. As the transmission probability depends on the electron spin orientation due to spin filtering effects as discussed above, the measurement of spin-dependent photocurrent (the difference $\Delta I$ between the photocurrents for excitations by right- and left-handed circularly polarized light) provides information about spin-dependent transport from the GaAs into the ferromagnets across the interface.

\section{Experimental studies of spin transport into ferromagnets}

A pioneering study of electron spin transport using optical spin orientation was reported by Prins et al. [16]. Since that first experiment, there have been many related studies [16,24-26,75-86]. Taniyama et al. [78], Steinmuller et al. [79] and Park et al. [86] measured the spin-dependent photocurrent for $\mathrm{Fe} / \mathrm{AlO}_{x} / \mathrm{GaAs}, \mathrm{NiFe}(\mathrm{Fe}) / \mathrm{GaAs}$ and $\mathrm{Fe} / \mathrm{MgO} / \mathrm{GaAs}$ interfaces, respectively, under forward bias with optical spin orientation. It was concluded in these studies that only magnetic circular dichroism arising from the difference in the absorption coefficients of right- and left-handed circularly polarized light was found, and that no spin filtering effect could be detected at large reverse bias [78,79]. Isakovic et al. [75] detected weakly spin polarized photocurrent signals across the Schottky interface of a device consisting of an InGaAs/GaAs QW and an FeCo layer. An interesting demonstration using an $\mathrm{Fe} / \mathrm{Tb}$ multilayer on an $\mathrm{MgO} / \mathrm{AlGaAs} / \mathrm{GaAs} \mathrm{QW}$ was also reported by Hövel et al. [76], where the $\mathrm{Fe} / \mathrm{Tb}$ multilayer exhibits spontaneous out-of-plane magnetization induced by interfacial perpendicular magnetic anisotropy. The spin-dependent photocurrent was measured in the remanent state. As 

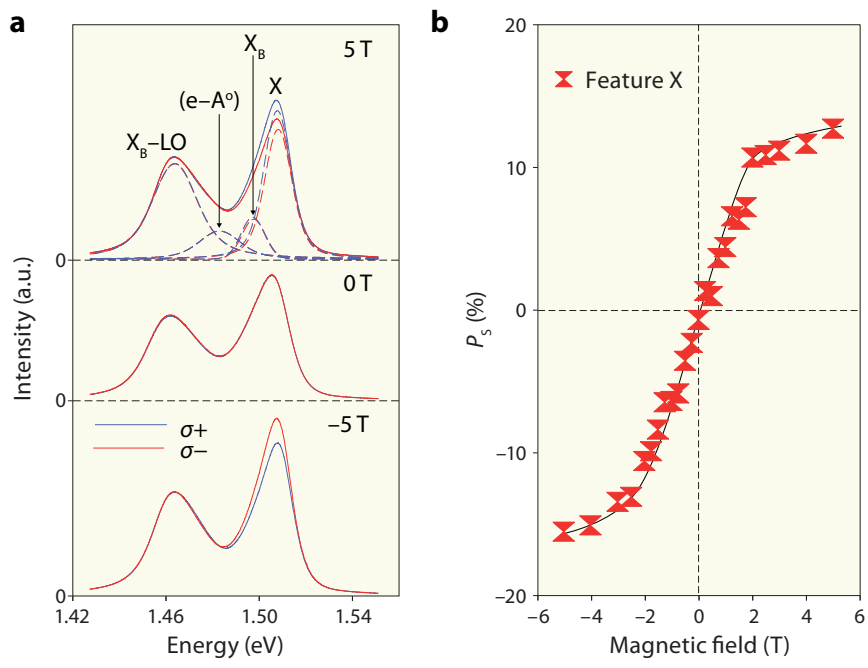

Figure 6. (a) Circularly polarized electroluminescence spectra for an Fe/GaAs QW spin-LED structure. (b) Magnetic field dependence of spin polarization for feature X. Modified after Ref. 26 (๔ 2010 APS).

the spin polarization of electrons generated by optical spin orientation is strongly dependent on the energy of the circularly polarized light due to simultaneous excitations from the split-off subband at $\Gamma_{7}$, tuning of the energy of light is critical to investigating the spin-dependent photocurrent across the interface. Kurebayashi et al. [80] reported the inversion of sign in the spin-dependent photocurrent signal associated with the spin filtering effect upon varying the excitation energy. However, the values of spin-dependent photocurrent reported so far are at the few percent level, in clear contrast with the large spin polarization estimated from circular polarized electroluminescence as given in the previous section.

Recently, Renucci et al. [84] carefully studied spin-dependent photocurrent in ferromagnetic metal/AlGaAs/GaAs QW heterostructures with $\mathrm{Al}_{2} \mathrm{O}_{3}$ or $\mathrm{MgO}$ tunnel barriers, in combination with measurements of the circular polarization of electroluminescence under spin injection conditions and time-resolved photoluminescence for the same devices. From the combined results, they concluded that the weak polarization of the photocurrent for reverse and zero bias is a consequence of the Zeeman splitting of the QW excitons, which yields different absorption coefficients for circular polarized excitation light with different helicities. They also discussed the reason for the relatively small spin-dependent photocurrent, suggesting that the key factor for obtaining a large spin-dependent photocurrent is that the photogenerated minority-spin electrons reflected by the ferromagnetic layer must recombine with holes in the semiconductor. Otherwise, the same flow of minority and majority electrons is obtained, giving rise to a vanishing spin-dependent photocurrent.

Wada et al. [26] reported on spin-dependent photocurrent across an Fe/AlGaAs/GaAs QW interface using a combined approach of electroluminescence and the optical spin orientation method. Figure 6(a) shows the results of optical polarization-resolved electroluminescence from a GaAs QW under spin injection from an iron layer. A clear splitting of the right- and left-handed circularly polarized components can be seen for sub-feature X due to the recombination of free excitons in the QW, as similarly observed by Mallory et al. [46]. Using the accurate information on the band structure of the QW thus obtained, the photon energy for optical spin orientation experiments can be determined, allowing free excitons with large spin polarization to be generated in the QW. Shown in Figure $7(\mathrm{a})$ is the variation in spin-dependent photocurrent $(\Delta I)$ as a function of bias voltage applied across the interface. A dip appears in the spin-dependent photocurrent at -0.058 and $-0.179 \mathrm{eV}$ for excitation energies of 1.503 and $1.666 \mathrm{eV}$, respectively, where $1.503 \mathrm{eV}$ is the energy of free excitons in the GaAs QW. The spin-dependent
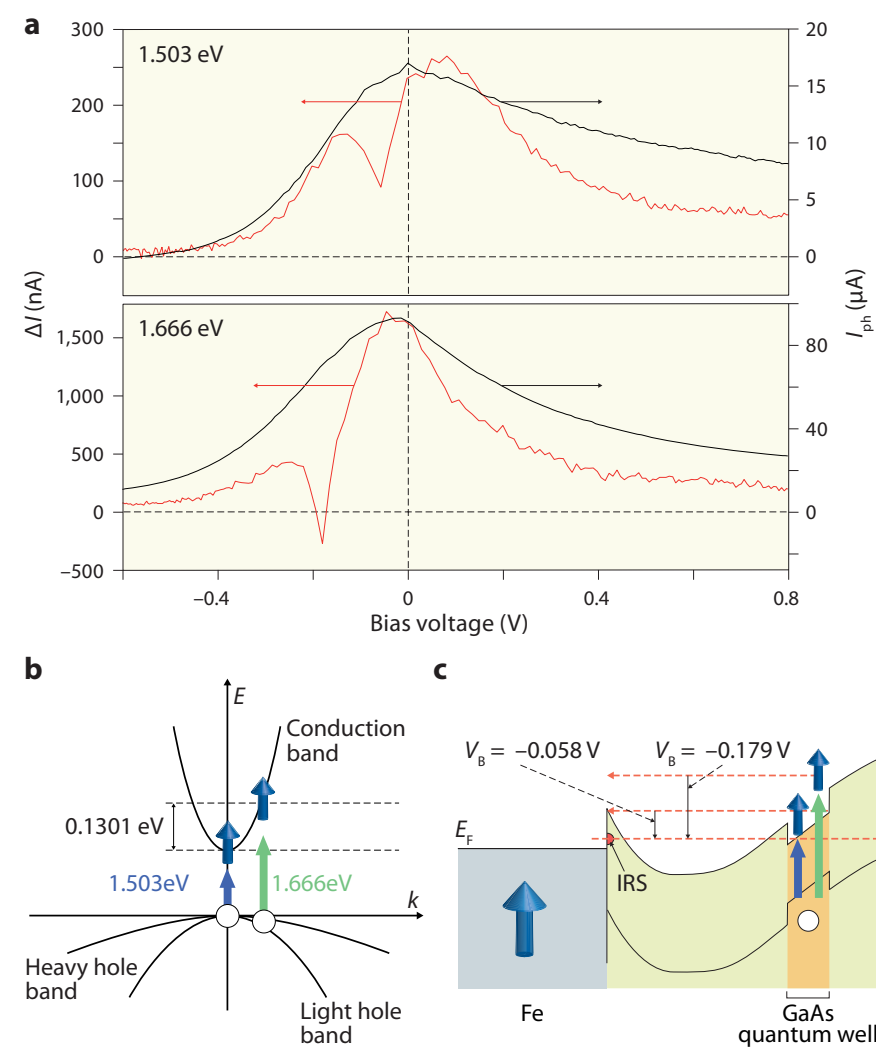

C

Figure 7. (a) Spin-dependent photocurrent $(\Delta l)$ and spin-independent photocurrent $\left(I_{\text {ph }}\right)$ as a function of bias voltage. (b) Electron excitation processes for 1.503 and $1.666 \mathrm{eV}$ excitations. At $1.503 \mathrm{eV}$, spin-polarized electrons are generated at the $\Gamma$ point of the GaAs QW. At $1.666 \mathrm{eV}$, non-thermal equilibrium electrons or hot electrons are generated, so excited electrons have a $\boldsymbol{k}$ vector corresponding to the energy gap of $1.666 \mathrm{eV}$. The heavy-hole and light-hole subbands split and the excitation from the heavy-hole subband is dominant in this case, resulting in excited electrons at $0.1301 \mathrm{eV}$ above the conduction band minimum. (c) Schematic diagram of resonant tunneling process via localized states at the interface for 1.503 and $1.666 \mathrm{eV}$ excitations. The energy levels of the photogenerated spins at 1.503 and $1.666 \mathrm{eV}$ match the localized state as bias voltages $\left(V_{\mathrm{B}}\right)$ of -0.058 and $-0.179 \mathrm{~V}$ are applied at the interface, respectively. Modified after Ref. 26 (๔ 2010 APS).

photocurrent is reversed by switching the magnetic field, indicating that the features correspond fully to spin-dependent signals. Such dip features do not appear in the corresponding spin-independent photocurrent $\left(I_{\mathrm{ph}}\right)$. The mechanism of the dip in $\Delta I$ can be interpreted in terms of a Breit-Wigner resonant tunneling process via localized states, as depicted in Figure 7(c) [87]. Although the detail is beyond the scope of this review, the spin-dependent photocurrent can be expressed by a negative $-4 P_{\mathrm{A}} P_{\mathrm{F}} /\left(1-P_{\mathrm{F}}^{2}\right)<0$ at resonance, while that at off-resonance is given by a positive $4 P_{\mathrm{A}} P_{\mathrm{F}}>0$, where $P_{\mathrm{F}}$ and $P_{\mathrm{A}}$ are the spin polarizations of electrons in iron and $\mathrm{AlGaAs}$, respectively.

These demonstrations show that the optical spin orientation method is a very powerful means of obtaining insights into the mechanism of spin-dependent electron transport across ferromagnet/semiconductor interfaces. These demonstrations also prove that the spin filtering effect can convert spin information in a semiconductor into a pure current value, making it possible to develop 'spin detectors' suitable for direct integration into electronic devices.

\section{Prospects for spintronics technology}

We reviewed selected topics on electrical and optical spin injection into semiconductors and electron spin transport processes across the ferromagnet/semiconductor interface. The review shows that the efficiency of spin injection can be much improved by engineering 
ferromagnet/semiconductor interfaces, as demonstrated in many experimental studies using Schottky barriers, nonmagnetic insulating barriers and magnetic insulating spin filters. Recent studies of spin-polarized electron transport across ferromagnet/semiconductor interfaces under optical spin orientation have also shown that a better understanding of the spin filtering effect could open a path for the creation of spin-detection technology for direct use in electronic devices, although such technology is still in an early pioneering stage. In order to make the design of spintronic devices a reality, not only spin injection and detection but also other technologies, such as control of the magnetization orientation of a source ferromagnet, are of importance. Achieving this functionality with low energy consumption is of particular significance. Studies of ferromagnet/ferroelectric heterostructures may offer a promising basis for the control of magnetization orientation by manipulating the interface-sensitive magnetic properties that arise from strain and/or electronic modification of the ferromagnet $[88,89]$. As the utilization of ferroelectrics or piezoelectrics can transfer strain via the interface by applying an electric field without current flow, such heterostructured devices have stimulated much interest in this rapidly growing field. In addition, in order to be incorporated into existing processes, carefully considered overall architectures of spintronic devices are indeed needed, although such a prototype spin injection-based device has yet to be demonstrated.

Nevertheless, updated visions for possible applications of spin injection have already been reported by several groups. One application is a spin-based insulated gate FET proposed by Hall and Flatté [4], who performed a careful quantitative comparison of the key elements of transistor power dissipation between spin-based insulated gate FETs and charge-based metal-oxide-semiconductor FETs. They demonstrated that a sufficiently small spin-based transistor could surpass the performance of charge-based transistors at room temperature. In demonstrating a spin-injected FET in a high-mobility InAs heterostructure, Koo et al. [5] observed an oscillatory conductance as a function of gate voltage, enabling the manipulation of injected electron spins. Another technology that is basically ready for practical use is the reduced threshold current offered by a vertical-cavity surface-emitting laser by pumping with spin-polarized electrons [90]. These applications offer the promise of spintronic devices with potential advantages in power consumption. The 2009 assessment of spin transistors in the International Technology Roadmap for Semiconductors [91] is also significantly better in several criteria compared with the assessments reported in the 2005 and 2007 roadmaps. This trend is now very encouraging and we envisage that further work will overcome the challenging issues of spin injection and detection, leading toward the integration of this technology and incorporation into commercial electronic devices.

\section{Acknowledgments}

The authors would like to acknowledge the financial support of the Precursory Research for Embryonic Science and Technology (PRESTO) program of the Japan Science and Technology Agency, the Industrial Technology Research Grant program (2009) of the New Energy and Industrial Technology Development Organization (NEDO), Japan, Grants-in-Aid for Scientific Research (Nos 18686050 and 22360265) from the Ministry of Education, Culture, Sports, Science and Technology, Japan, the Mitsubishi Foundation, the Shimadzu Science Foundation, the Kato Science Foundation, the Nippon Sheet Glass Foundation for Materials Science and Engineering, the Iketani Science and Technology Foundation and the Toray Science Foundation.

\section{References}

S. Datta, B. Das, Appl. Phys. Lett. 56, 665 (1990).

M. Johnson, J. Magn. Magn. Mater. 156, 321 (1996).

S. Sugahara, M. Tanaka, Appl. Phys. Lett. 84, 2307 (2004).

K. C. Hall, M. E. Flatté, Appl. Phys. Lett. 88, 162503 (2006).

H. C. Koo et al., Science 325, 1515 (2009).
R. Fiederling et al., Nature 402, 787 (1999).

Y. Ohno et al., Nature 402, 790 (1999).

J. C. Slonczewski, J. Magn. Magn. Mater. 159, L1 (1996).

E. Chen et al., IEEE Trans. Magn. 46, 1873 (2010).

Y. A. Bychkov, E. I. Rashba, J. Phys. C 17, 6039 (1984).

1. F. Meier, B. P. Zakharchnya, Optical Orientation (North-Holland,

Netherlands, 1984).

M. W. Wu, J. H. Jiang, M. Q. Weng, Phys. Rep. 493, 61 (2010).

13. B. T. Jonker, S. C. Erwin, A. Petrou, A. G. Petukhov, MRS Bulletin 28, 740 (2003).

14. F. J. Jedema, A. T. Filip, B. J. van Wees, Nature 410, 345 (2001).

15. M. Johnson, R. H. Silsbee, Phys. Rev. Lett. 55, 1790 (1985).

16. M. W. J. Prins et al., J. Phys-Condens. Mat. 7, 9447 (1995).

17. P. Chen et al., Phys. Rev. B 74, 241302(R) (2006).

18. G. Schmidt, J. Phys. D 38, R105 (2005).

19. R. A. de Groot, F. M. Mueller, P. G. van Engen, K. H. J. Buschow, Phys. Rev. Lett. 50, 2024 (1983)

20. C. Palmstrøm, MRS Bulletin 28, 725 (2003).

21. J. M. D. Coey, C. L. Chien, MRS Bulletin 28, 720 (2003).

22. J. S. Moodera, T. S. Santos, T. Nagahama, J. Phys-Condens. Matt. 19, 165202 (2007).

23. D. T. Pierce, F. Meier, Phys. Rev. B 13, 5484 (1976).

24. E. Wada et al., Appl. Phys. Lett. 96, 102510 (2010).

25. E. Wada et al., Appl. Phys. Lett. 97, 172509 (2010).

26. E. Wada et al., Phys. Rev. Lett. 105, 156601 (2010).

27. M. J. Howes, D. V. Morgan, Gallium Arsenide (John Wiley \& Sons, USA, 1985).

28. M. I. D’yakonov, V. I. Perel', Zh. Eksp. Toer. Fiz. 60, 1954 (1971).

29. G. L. Bir, A. G. Aronov, G. E. Pikus, Zh. Eksp. Toer. Fiz. 69, 1382 (1975).

30. R. J. Elliott, Phys. Rev. 96, 266 (1954).

31. Y. Yafet, Solid State Physics 14 (Academic Press, USA, 1963).

32. M. I. D’yakonov, V. I. Perel', Zh. Eksp. Toer. Fiz. 38, 362 (1973).

33. A. Kawaharazuka et al., Appl. Phys. Lett. 85, 3492 (2004).

34. P. C. van Son, H. van Kempen, P. Wyder, Phys. Rev. Lett. 58, 2271 (1987).

35. M. Johnson, R. H. Silsbee, Phys. Rev. B 35, 4959 (1987).

36. T. Inouchi, T. Marukame, M. Ishikawa, H. Sugiyama, Y. Saito, Appl. Phys. Exp. 2, 023006 (2009).

37. X. Lou et al., Nature Phys. 3, 197 (2007).

38. I. Appelbaum, B. Huang, D. J. Monsma, Nature 447, 295 (2007).

39. O. M. J. van't Erve et al., Appl. Phys. Lett. 91, 212109 (2007).

40. S. P. Dash, S. Sharma, R. S. Patel, M. P. de Jong, R. Jansen, Nature 462, 491 (2009).

41. T. Sasaki et al., IEEE Trans. Magn. 46, 1436 (2010).

42. H. J. Zhu et al., Phys. Rev. Lett. 87, 016601 (2001).

43. B. T. Jonker et al., Phys. Rev. B 62, 8180 (2000).

44. A. T. Hanbicki et al., Appl. Phys. Lett. 82, 4092 (2003).

45. C. H. Li et al., Appl. Phys. Lett. 91, 262504 (2007).

46. R. Mallory et al., Phys. Rev. B 73, 115308 (2006).

47. B. T. Jonker et al., Appl. Phys. Lett. 79, 3098 (2001).

48. M. C. Hickey et al., Phys. Rev. B 75, 193204 (2007).

49. C. Adelmann, X. Lou, J. Strand, C. J. Palmstrøm, P. A. Crowell, Phys. Rev. B 71, 121301 (2005).

50. E. Oh et al., J. Appl. Phys. 106, 043515 (2009).

51. A. Sinsarp, T. Manago, F. Takano, H. Akinaga, Jap. J. Appl. Phys. 46, L4 (2007).

52. T. Manago, H. Akinaga, Appl. Phys. Lett. 81, 694 (2002).

53. T. Manago, A. Sinsarp, H. Akinaga, J. Appl. Phys. 102, 083914 (2007).

54. O. M. J. van't Erve et al., Appl. Phys. Lett. 84, 4334 (2004).

55. H. Saito et al., Appl. Phys. Lett. 96, 012501 (2010).

56. X. Jiang et al., Phys. Rev. Lett. 94, 056601 (2005).

57. G. Salis et al., Appl. Phys. Lett. 87, 262503 (2005).

58. X. Y. Dong et al., Appl. Phys. Lett. 86, 102107 (2005).

59. M. Ramsteiner et al., Phys. Rev. B 78, 121303 (2008).

60. M. C. Hickey et al., Appl. Phys. Lett. 86, 252106 (2005).

61. M. C. Hickey et al., Appl. Phys. Lett. 92, 232101 (2008).

62. D. Damsgaard et al., J. Appl. Phys. 105, 124502 (2009).

63. V. F. Motsnyi et al., Phys. Rev. B 68, 245319 (2003).

64. B. T. Jonker, G. Kioseoglou, A. T. Hanbicki, C. H. Li, P. E. Thompson, Nature Phys. 3, 542 (2007).

65. R. Jansen et al., Phys. Rev. B 82, 241305(R) (2010).

66. G. Schmidt, D. Ferrand, L. W. Molenkamp, A. T. Filip, B. J. van Wees, Phys. Rev. B 62, R4790 (2000).

67. E. I. Rashba, Phys. Rev. B 62, R16267 (2000).

68. K. Hamaya et al., Phys. Rev. Lett. 102, 137204 (2009).

69. P. A. Dowben, R. Skomski, J. Appl. Phys. 95, 7453 (2004).

70. J. J. Attema et al., J. Phys-Condens. Matt. 16, S5517 (2004).

71. T. S. Santos, J. S. Moodera, Phys. Rev. B 69, 241203(R) (2004).

72. A. T. Filip et al., Appl. Phys. Lett. 81, 1815 (2002). 
73. T. Nagahama,T. S. Santos, J. S. Moodera, Phys. Rev. Lett. 99, 016602 (2007).

74. G. Lampel, Phys. Rev. Lett. 20, 491 (1968).

75. A. F. Isakovic et al., Phys. Rev. B 64, 161304 (2001).

76. S. Hövel et al., Appl. Phys. Lett. 92, 242102 (2008).

77. A. Hirohata et al., Phys. Rev. B 66, 035330 (2002).

78. T. Taniyama, G. Wastlbauer, A. Ionescu, M. Tselepi, J. A. C. Bland, Phys. Rev. B 68, $134430(2003)$

79. S. J. Steinmuller, C. M. Gürtler, G. Wastlbauer, J. A. C. Bland, Phys. Rev. B 72, 045301 (2005).

80. H. Kurebayashi et al., Appl. Phys. Lett. 96, 022505 (2010).

81. H. Kurebayashi et al., Appl. Phys. Lett. 91, 102114 (2007).

82. S. E. Andresen et al., Phys. Rev. B 68, 073303 (2003).
83. S. Isber, Y. J. Park, J. S. Moodera, D. Heiman, J. Appl. Phys. 103, 07 D713 (2008).

84. P. Renucci et al., Phys. Rev. B 82, 195317 (2010).

85. C. Shen et al., Appl. Phys. Lett. 97, 162104 (2010).

86. Y. J. Park et al., J. Phys-Condens. Matt. 23, 116002 (2011).

87. S. Datta, Electronic Transport in Mesoscopic Systems (Cambridge University Press, UK, 1995).

88. C. A. F. Vaz, J. Hoffman, C. H. Ahn, R. Ramesh, Adv. Mater. 22, 2900 (2010).

89. Y. Shirahata et al., Appl. Phys. Lett. 99, 022501 (2011).

90. J. Rudolph, D. Hägele, H. M. Gibbs, G. Khitrova, M. Oestreich, Appl. Phys. Lett. 82, 4516 (2003).

91. International Technology Roadmap for Semiconductors (Semiconductor Industry Association, San Jose, CA, USA, 2009). http://public.itrs.net

\section{Author profiles}

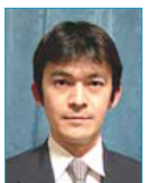

\section{Tomoyasu Taniyama}

Tomoyasu Taniyama is an associate professor in the Materials and Structures Laboratory at the Tokyo Institute of Technology, Japan. He obtained his PhD degree from Keio University, Japan, in 1997 for his work on the magnetic properties of transition metal nanoparticles. He joined the National Research Institute for Metals to pursue research on the magnetotransport properties of lithographically defined magnetic nanostructures. He moved to the Tokyo Institute of Technology in 1998. During a two-year stay at the Cavendish Laboratory of the University of Cambridge, UK, from 2001 to 2003, he studied the transmission of optically excited spin-polarized electrons across ferromagnetic metal/semiconductor interfaces. His research is now focused on the physical properties of spin electronic devices, in particular spin injection, spin detection and spin manipulation in magnetic heterostructures.

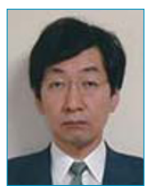

\section{Mitsuru Itoh}

Mitsuru Itoh is a professor in the Materials and Strictures Laboratory of the Tokyo Institute of Technology, Japan. He received his master and doctor degrees from the Tokyo Institute of Technology in 1979 and 1982, and his Bachelor of Engineering degree from the Nagoya Institute of Technology, Japan, in 1977. He started his research career in the Faculty of Engineering of Osaka University as an assistant professor in 1982, where he worked on solid-state chemical sensors. He became an associate professor of the Materials and Strictures Laboratory in 1988 where he continues his study of solid-state chemistry on inorganic materials. His research interests include the structure-property relationships of oxide materials, especially in the synthesis of novel materials with interesting electronic, dielectric, magnetic and ion-conducting properties.

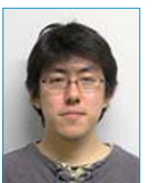

\section{Eiji Wada}

Eiji Wada received his PhD degree from the Tokyo Institute of Technology in 2010 for optical studies of electron spin transmission in ferromagnet/semiconductor heterostructures. He worked as a postdoctoral researcher at the Materials and Structures Laboratory of the Tokyo Institute of Technology and moved to the Institute of Scientific and Industrial Research at Osaka University in 2011. He worked in the field of spintronics for seven years investigating the electron spin transmission processes at ferromagnets/semiconductor interfaces using a combined approach of optical spin orientation and polarized electroluminescence. His current research interests include the quantum spin Hall effect in topological insulators.

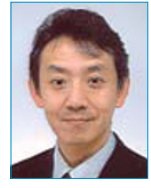

\section{Masahito Yamaguchi}

Masahito Yamaguchi has worked in III-V semiconductors for over 15 years, investigating the optical and electronic properties of the nanostructures in the Nagoya University, Japan. Recently, he has been interested in growth mechanisms with molecular beam epitaxy and/or metal organic vapor phase epitaxy. He is also investigating nanowires of GaAs and GaN in order to develop novel optical and electronic devices. He received his PhD from the Osaka University, Japan, in 1995 for research on Wannier-Stark effect in III-V semiconductor superlattices. 